\title{
A roadmap from unknowns to knowns: Advancing our understanding of the microbiomes of commercially available tobacco products
}

\author{
Suhana Chattopadhyay ${ }^{1} \cdot$ Leena Malayil $^{1} \cdot$ Emmanuel F. Mongodin $^{2,3} \cdot$ Amy R. Sapkota $^{1}$ (D) \\ Received: 14 December 2020 / Revised: 10 February 2021 / Accepted: 14 February 2021 / Published online: 11 March 2021 \\ (C) The Author(s), under exclusive licence to Springer-Verlag GmbH, DE part of Springer Nature 2021
}

\begin{abstract}
Tobacco smoking is still the leading cause of preventable diseases and death in the USA and throughout the globe. Under Section 904(a)(3) of the US Federal Food, Drug, and Cosmetic Act, tobacco manufacturing companies need to report on quantities of harmful and potentially harmful constituents (HPHCs) in all tobacco products. While the extensive HPHC list of 2012 includes 93 chemicals, which are categorized as carcinogenic, respiratory, cardiovascular, or reproductive toxicants or addictive compounds, it fails to include microorganisms (bacteria and fungi) that have been shown to contribute to adverse health outcomes among tobacco users. Nevertheless, over the last 50 years, researchers have studied microorganisms in a variety of tobacco products using both culture-based and culture-independent techniques. In this mini-review, we provide an overview of this body of research, detailing the bacterial and fungal microbiomes residing in commercial tobacco products. Overall, studies have characterized over 89 unique bacterial genera and 19 fungal genera in cigarettes, cigars, cigarillos, hookah, and smokeless tobacco. The most predominant bacterial genera are Bacillus, Pseudomonas, and Staphylococcus. Fungal genera identified have included Aspergillus, Penicillium, Mucor, Alternaria, Cladosporium, Streptomyces, and Candida, to name a few. While some of the identified microorganisms are known human pathogens, others are potential opportunistic pathogens. Given the vast array of microorganisms that are present across diverse types of tobacco products, future research should be focused on the viability of these microorganisms, as well as their ability to transfer to the user's respiratory tract, potentially contributing to adverse health outcomes.
\end{abstract}

\section{Key points}

- Commercial tobacco products harbor diverse bacterial and fungal communities.

- Some of these microorganisms are known or opportunistic human pathogens.

- Research on their viability and transmission to users' respiratory tracts is needed.

Keywords Tobacco $\cdot$ Cigarettes $\cdot$ Microbiome $\cdot$ Bacteria $\cdot$ Fungi

Suhana Chattopadhyay and Leena Malayil shares co-first authorship.

Disclaimer Dr. Mongodin contributed to this article as an employee of the University of Maryland School of Medicine. The views expressed are his own and do not necessarily represent the views of the National Institutes of Health or the United States Government

Amy R. Sapkota

ars@umd.edu

1 Maryland Institute for Applied Environmental Health, University of Maryland School of Public Health, College Park, MD 20742, USA

2 Institute for Genome Sciences and Department of Microbiology and Immunology, University of Maryland School of Medicine, Baltimore, MD 21201, USA

3 Present address: Division of Lung Diseases, National Institutes of Health (NIH), National Heart, Lung and Blood Institute (NHLBI), Bethesda, MD, USA

\section{Introduction}

Tobacco smoking remains the leading cause of preventable disease and death (Healthy People 2020). According to the Centers for Disease Control and Prevention's Morbidity and Mortality Report, about 49.1 million American adults in 2018 used some sort of tobacco products (Creamer 2019), including any combustible product (41.2 million), electronic cigarettes (e-cigarettes) (8.1 million), cigars (9.6 million), and smokeless tobacco (5.9 million). As a result, tobacco use remains a major public health concern and is responsible for approximately 7 million deaths worldwide each year (WHO 2017). In the United States, approximately 480,000 deaths per year are due to cigarette smoking and secondhand smoke (CDC 
Tobacco Free 2018). Tobacco companies regularly modify constituents of their existing products (e.g., nicotine concentrations, flavoring, additives) (Kreslake et al. 2008; Kostygina et al. 2016) and introduce novel products (such as the new generation of electronic nicotine delivery systems) to the market to appeal to and attract users (de Andrade et al. 2013). Tobacco products are available in various forms: (1) traditional combustible products including cigarettes and cigars; (2) water pipe/hookah; (3) noncombustible smokeless tobacco (like snuff, snus, etc.); and (4) other electronic nicotine delivery systems such as electronic cigarettes (e-cigarettes).

Tobacco products, irrespective of their production method, typically are known to contain over 4000 chemicals (Konstantinou et al. 2018), including nicotine, carcinogens, and other toxicants, many of which play a key role in the development of adverse health effects among users (Konstantinou et al. 2018). In 2012, under the Food, Drug, and Cosmetic Act (FD\&C Act), the US Food and Drug Administration (U.S. FDA 2020a) established a list of 93 harmful and potentially harmful constituents (HPHC) in tobacco and tobacco smoke. Tobacco manufacturing companies must comply with this act and report on quantities of these HPHCs in all tobacco products. The HPHC list comprises chemicals that are categorized as carcinogens (or potential carcinogens), as well as respiratory, cardiovascular, reproductive, and developmental toxicants. However, the list is ignoring an entire group of tobacco constituents that could be critically important with regard to users' health: microorganisms.

Tobacco, being an agricultural product, is rich in microorganisms that naturally colonize the plants. In addition, these microorganisms are key to curing tobacco, a process necessary to prepare tobacco leaves for consumption. Tobacco users are, therefore, chronically exposed to these microorganisms as they use tobacco products. And yet, microbiological constituents of tobacco, as well as their potential associated health impacts, have received little scientific or regulatory attention in the past 50 years despite multiple studies demonstrating their presence across all tobacco products.

This mini-review aims to provide a broad overview of the studies conducted over the past 50 years (1970-2020) that have focused on the microbiological constituents in combustible tobacco products (cigarettes, little cigars, and cigarillos), water pipe tobacco/hookah, and smokeless tobacco products (Fig. 1). We summarize the vast array of bacterial and fungal communities that reside within these commercially available tobacco products, as well as the effect of additives and flavors on these communities, without claiming to put forth an exhaustive compilation. Additionally, we address the current gaps in this body of literature and provide a glimpse into future studies that are required to better understand the microbiome of tobacco products, especially newer products on the market such as e-cigarettes that claim to be less harmful to users.

\section{Bacterial constituents of tobacco products}

\section{Combustible tobacco products: cigarettes, little cigars, and cigarillos}

The most prevalent traditional combustible tobacco products include cigarettes, little cigars, and cigarillos. Irrespective of the differences in their size, shape, and appearance, all of these products have been shown to harbor diverse bacterial species, including human pathogens (Table 1). Using culture-based approaches, early studies identified species of Actinomycetes (Kurup et al. 1983), Erwinia (Larsson et al. 2008), Bacillus (Rooney et al. 2005), Kurthia (Rooney et al. 2005), and Mycobacterium (Eaton et al. 1995) in tobacco particles, smoked filters, and cigarette filters (Eaton et al. 1995).

Due to the very small percentage of bacteria that can be cultured in the laboratory, culture-based approaches are limited in determining the vast array of microorganisms present in tobacco products. One of the earliest studies to focus on culture-independent techniques to identify bacterial species in commercially available cigarette products was by Sapkota et al. (2010). In this study, a 16S rRNA gene-based taxonomic microarray, along with cloning and sequencing, were used to identify bacteria, including Acinetobacter, Bacillus, Burkholderia, Clostridium, Klebsiella, Amaracoccus, Legionellales, Methylobacterium, Nostoc, Paracoccus, Pseudomonas aeruginosa, P. chlororaphis, P. cichorii, and Serratia, in most of the tested cigarette products (Sapkota et al. 2010). Other potentially pathogenic bacteria detected included Campylobacter, Enterococcus, Proteus, and Staphylococcus.

Further studies based on next-generation sequencing from multiple groups have revealed the presence of more than 89 unique bacterial genera in commercial cigarettes (Table 1). The top three bacterial phyla identified across all of these studies are Proteobacteria, Actinobacteria, and Firmicutes, and the most predominant bacterial genera are Bacillus, Pseudomonas, and Staphylococcus. Since some of the species within these genera are also known opportunistic pathogens, their high relative abundance in cigarettes could be a cause of potential health concern among smokers. For example, Pseudomonas species have been associated with chronic lung infections and cystic fibrosis among smokers (Erb-Downward et al. 2011; Fodor et al. 2012). Recently, Wu et al. (2018) demonstrated that nicotine, an active component in cigarettes, enhances biofilm formation in Staphylococcus epidermis (a common bacterial colonizer of the human skin and mucous membranes) (Wu et al. 2018). S. epidermis has also been identified in cigarettes by Sapkota et al. (2010) and has been associated with nosocomial infections in recent years (Götz 2002; Zschiedrich et al. 2016). Multiple Bacillus species (B. pumilus, B. cereus, and B. subtilis) have been identified in commercial cigarettes by several researchers (Rooney et al. 
Fig. 1 Schematic representation of microbial (bacteria and fungi) communities residing within commercially available tobacco products

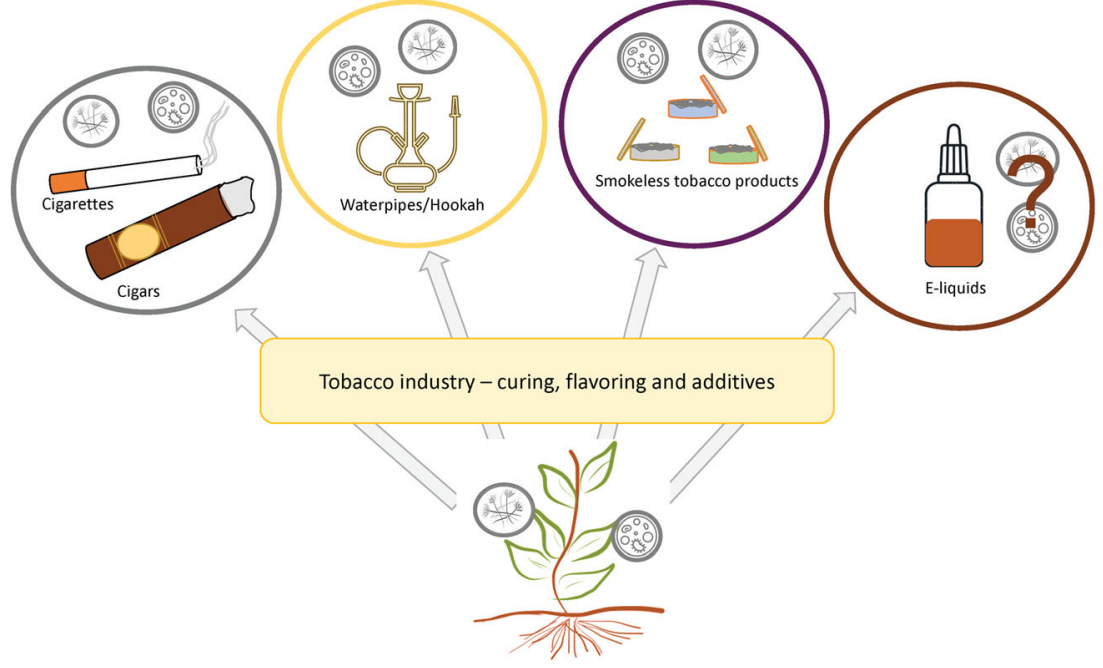

2005; Sapkota et al. 2010; Chopyk et al. 2017a, b; Malayil et al. 2020), which have also been associated with respiratory infections and pneumonia among smokers (Rooney et al. 2005). Apart from human pathogens, thermophiles such as Anoxybacillus, Schlegella, and Silanimonas have also been identified in cigarette tobacco (Chopyk et al. 2017a). The presence of these thermophiles raises concerns given their ability to withstand high temperatures such as those taking place during cigarette combustion.

Sequencing-based studies also have revealed that the differences in bacterial community composition across combustible tobacco products are dependent on the specific brands, flavors, and lots (Chopyk et al. 2017b; Malayil et al. 2020). Chopyk et al. (2017a) demonstrated significant differences in bacterial community composition between cigarette brands (Camel and Newport), with Pseudomonas, Bacillus, and Pantoea showing the highest relative abundance in all samples tested. Moreover, core microbiomes of more than 15 bacterial operational taxonomic units (OTUs) were identified for each cigarette brand, while 11 OTUs were shared among all brands irrespective of incubation condition (Chopyk et al. 2017a). Similar results were found between the top two selling U.S. brands (Newport and Marlboro) of cigarettes (Malayil et al. 2020), which are the most popular among adolescents and young adults (CDC Tobacco Free 2020). Along with significant differences in bacterial communities between the two brands, Malayil et al. (2020) also showed significant differences between the two varieties of mentholated Newport cigarettes (menthol box and menthol gold). It is noteworthy that all of the abovementioned studies evaluated bacterial communities residing within commercially available cigarettes. Yet, since the chemical constituents of these products vary across products, it is almost impossible to assess the effects of specific chemical components, such as nicotine and menthol, on the bacterial communities of commercial products.
This knowledge gap is critical, as these two chemicals have been shown to significantly affect multiple bacterial species. For example, nicotine has been shown to stimulate Streptococcus gordonii planktonic cell growth, biofilm formation, and gene expression of binding proteins, all of which could contribute to subsequent attachment of pathogens to tooth surfaces and development of periodontal disease in cigarette smokers (Huang et al. 2014). On the other hand, menthol has been shown to possess antibacterial activity against Gram-positive and Gram-negative bacteria as well as fungal species (Schelz et al. 2006; Shapira and Mimran 2007; Patel et al. 2007) although bacterial adaptation to menthol has been observed (Landau and Shapira 2012). Ongoing work in our group is addressing these issues by evaluating the impact of differing nicotine and menthol levels on the bacterial communities of SPECTRUM research cigarettes, for which levels of these constituents are known.

Given the vast array of bacterial species residing in combustible tobacco products and the established fact of the negative effects of smoking on user's health, two major questions arise to bridge a potential causal relationship between tobacco bacterial communities and adverse health effects among users. (1) Are these tobacco-associated bacteria viable? And (2) are the bacteria originating from the unburnt region of cigarettes/cigars during smoking able to be transferred to users via mainstream smoke and contribute to oral and lung microbiome dysbiosis, potentially impacting the users' health? Previous studies have demonstrated the presence of viable bacteria in the filters of smoked cigarettes (e.g., Mycobacterium avium) (Eaton et al. 1995), as well as the presence of other microbial constituents, including lipopolysaccharides, peptidoglycans, and fungal biomass in mainstream smoke (Pauly and Paszkiewicz 2011). These data suggest that bacteria and fungi can survive the temperatures and gases produced 
Table 1 A list of studies describing bacterial profiles in tobacco products

\begin{tabular}{|c|c|c|c|}
\hline Phylum & Genus & Species & Studies, products tested, and methods \\
\hline \multirow[t]{2}{*}{ Actinobacteria } & Actinomycetes & & $\begin{array}{l}\text { Kurup et al. (1983) (commercial cigarettes) - culture; Larsson et al. (2008) (commer- } \\
\text { cial cigarettes) - culture }\end{array}$ \\
\hline & Bifidobacterium & & Chopyk et al. $(2017 \mathrm{a}, \mathrm{b})$ (commercial cigarettes)—sequencing \\
\hline Actinobacteria & Brachybacterium & & Chattopadhyay et al. (2019) (little cigars and wrappers) - sequencing \\
\hline Actinobacteria & Brevibacterium & B. aureum & Malayil et al. (2020) (commercial tobacco) sequencing \\
\hline \multirow[t]{2}{*}{ Actinobacteria } & Corynebacterium & C. xerosis & Sapkota et al. (2010) (commercial cigarettes) - microarray, cloning, and sequencing \\
\hline & & C. stationis & $\begin{array}{l}\text { Malayil et al. (2020) (commercial cigarettes) - sequencing; } \\
\text { Chattopadhyay et al. (2019) (little cigars and cigarillos)—sequencing }\end{array}$ \\
\hline Actinobacteria & Atopobium & & Sapkota et al. (2010) (commercial cigarettes) - microarray, cloning, and sequencing \\
\hline Actinobacteria & Mycobacterium & M avium & Eaton et al. (1995) (commercial cigarettes and smoked cigarette filters) - culture \\
\hline Actinobacteria & Propionibacterium & P. acnes & Hani et al. (2018) (hookah)—sequencing \\
\hline Actinobacteria & Streptomyces & & $\begin{array}{l}\text { Smyth et al. (2017) (smokeless tobacco)—sequencing; Chopyk et al. (2017a, b) } \\
\text { (commercial tobacco)-sequencing }\end{array}$ \\
\hline Bacteroidetes & Barnesiella & & Hani et al. (2018) (hookah)—sequencing \\
\hline Bacteroidetes & Flavobacterium & & Hani et al. (2018) (hookah)—sequencing \\
\hline Candidatus & Puniceispirillum & & Tyx et al. (2020) (smokeless tobacco)—sequencing \\
\hline Cyanobacteria & Chlorogloeopsis & & Sapkota et al. (2010) (commercial cigarettes) - microarray, cloning, and sequencing \\
\hline Cyanobacteria & Nostoc & & Sapkota et al. (2010) (commercial cigarettes) - microarray, cloning, and sequencing \\
\hline Cyanobacteria & Lyngbya & & Sapkota et al. (2010) (commercial cigarettes) - microarray, cloning, and sequencing \\
\hline Cyanobacteria & Microcystis & & Sapkota et al. (2010) (commercial cigarettes) - microarray, cloning, and sequencing \\
\hline Cyanobacteria & Gloeothece & & Tyx et al. (2020) (smokeless tobacco)-sequencing \\
\hline \multirow{3}{*}{$\begin{array}{l}\text { Deinococcus- } \\
\text { Thermus }\end{array}$} & Deinococcus & & Chopyk et al. (2017a, b) (commercial cigarettes) - sequencing \\
\hline & Thermus & T. geothermalis & Chopyk et al. $(2017 \mathrm{a}, \mathrm{b})$ (commercial cigarettes) - sequencing \\
\hline & & T. scotoductus & Chopyk et al. 2017a, b(commercial cigarettes)—sequencing \\
\hline Firmicutes & Megasphaera & & Sapkota et al. (2010) (commercial cigarettes) - microarray, cloning, and sequencing \\
\hline Firmicutes & Aerococcus & & Chopyk et al. $(2017 \mathrm{a}, \mathrm{b})$ (commercial cigarettes)—sequencing \\
\hline Firmicutes & Anoxybacillus & A. flavithermus & Chopyk et al. $(2017 \mathrm{a}, \mathrm{b})$ (commercial cigarettes) — sequencing \\
\hline Firmicutes & Atopostipes & & Tyx et al. (2020) (smokeless tobacco)-sequencing \\
\hline Firmicutes & Atopococcus & A. tabaci & $\begin{array}{l}\text { Collins et al. (1992) (smokeless tobacco)—-sequencing; Chattopadhyay et al. (2019) } \\
\text { (little cigars and cigarillos)—sequencing }\end{array}$ \\
\hline \multirow[t]{7}{*}{ Firmicutes } & Bacillus & B. thuringiensis & $\begin{array}{l}\text { Kaelin and Gadani (2000) (cured tobacco leaves) - culture; Kaelin et al. (1994) } \\
\text { (cured tobacco leaves and dried tobacco residues) - culture }\end{array}$ \\
\hline & & B. cereus & $\begin{array}{l}\text { Rooney et al. (2005) (commercial cigarettes) - culture; Chopyk et al. (2017a, b) } \\
\text { (commercial cigarettes)-sequencing }\end{array}$ \\
\hline & & B. licheniformis & $\begin{array}{l}\text { Rubinstein and Pedersen (2002) (smokeless tobacco)—culture; Han et al. (2016) } \\
\text { (smokeless tobacco)—culture; Chopyk et al. (2017a, b) (commercial cigarettes)- } \\
\text { sequencing }\end{array}$ \\
\hline & & B. clausii & $\begin{array}{l}\text { Chopyk et al. (2017a, b) (commercial cigarettes)-sequencing; Malayil et al. (2020) } \\
\text { (commercial cigarettes)-sequencing; Chattopadhyay et al. (2019) } \\
\text { (little cigars and cigarillos)-sequencing }\end{array}$ \\
\hline & & B. coagulans & $\begin{array}{l}\text { Malayil et al. (2020) (commercial cigarettes)—sequencing; Chattopadhyay et al. } \\
\text { (2019) (little cigars and cigarillos)-sequencing }\end{array}$ \\
\hline & & B. novalis & Chopyk et al. $(2017 \mathrm{a}, \mathrm{b})$ (commercial cigarettes)—sequencing \\
\hline & & B. pumilus & $\begin{array}{l}\text { Rooney et al. (2005) (commercial cigarettes) — culture; Sapkota et al. (2010) (com- } \\
\text { mercial cigarettes)-microarray, cloning, and sequencing; Chopyk et al. (2017a, b) } \\
\text { (commercial cigarettes)-sequencing; Rubinstein and Pedersen (2002) } \\
\text { (smokeless tobacco) - culture; Han et al. (2016) (smokeless tobacco)-culture }\end{array}$ \\
\hline
\end{tabular}

B. thermoamylovorans Chattopadhyay et al. (2019) (little cigars wrapper)-sequencing 
Table 1 (continued)

\begin{tabular}{|c|c|c|c|}
\hline Phylum & Genus & Species & Studies, products tested, and methods \\
\hline & & B. flexus & Malayil et al. (2020) (commercial tobacco)—sequencing \\
\hline & & B. subtilis & $\begin{array}{l}\text { Rooney et al. (2005) (commercial cigarettes) - culture; Dygert (1957) } \\
\text { (smokeless tobacco) - culture; Rubinstein and Pedersen (2002) (smokeless tobac- } \\
\text { co)_culture; Han et al. (2016) (smokeless tobacco) - culture }\end{array}$ \\
\hline & & B. brevis & Rubinstein and Pedersen (2002) (smokless tobacco) — culture \\
\hline & & B. megaterium & Rubinstein and Pedersen (2002) (smokless tobacco) — culture \\
\hline & & B. safensis & Han et al. (2016) (smokeless tobacco) - culture \\
\hline Firmicutes & Carnobacterium & & $\begin{array}{l}\text { Han et al. (2016) (smokeless tobacco)_culture; Chattopadhyay et al. (2019) } \\
\text { (little cigars and cigarillos)—sequencing }\end{array}$ \\
\hline Firmicutes & Clostridium & & Sapkota et al. (2010) (commercial cigarettes) — microarray, cloning, and sequencing \\
\hline Firmicutes & Dialister & & Sapkota et al. (2010) (commercial cigarettes) — microarray, cloning, and sequencing \\
\hline Firmicutes & Desemzia & & Tyx et al. (2020) (smokeless tobacco) - sequencing \\
\hline \multirow[t]{2}{*}{ Firmicutes } & Enterococcus & E. cecorum & Chattopadhyay et al. (2019) (little cigars wrapper)—sequencing \\
\hline & & E. gallinarum & Sapkota et al. (2010) (commercial cigarettes)—microarray, cloning, and sequencing \\
\hline Firmicutes & Facklamia & & Chopyk et al. (2017a, b) (commercial cigarettes)—sequencing \\
\hline Firmicutes & Faecalibacterium & & Hani et al. (2018) (hookah)—sequencing \\
\hline Firmicutes & Geobacillus & & Han et al. (2016) (smokeless tobacco)—sequencing \\
\hline Firmicutes & Jeotgalicoccus & & Chopyk et al. (2017a, b) (commercial cigarettes)—sequencing \\
\hline Firmicutes & Kurthia & & $\begin{array}{l}\text { Rooney et al. (2005) (commercial cigarettes)-culture; } \\
\text { Smyth et al. (2017) (smokeless tobacco)-sequencing } \\
\text { Smyth et al. (2017) (smokeless tobacco)-sequencing } \\
\text { Chattopadhyay et al. (2019) (little cigars wrapper)-sequencing }\end{array}$ \\
\hline \multirow[t]{4}{*}{ Firmicutes } & Lactobacillus & L. plantarum & Chopyk et al. (2017a, b) (commercial cigarettes)—sequencing \\
\hline & & L. salivarius & Chattopadhyay et al. (2019) (little cigars wrapper)—sequencing \\
\hline & & L. delbrueckii & Smyth et al. (2017) (smokeless tobacco)—sequencing \\
\hline & & L. agilis & Chattopadhyay et al. (2019) (little cigars wrapper)—sequencing \\
\hline Firmicutes & Lentibacillus & & Chattopadhyay et al. (2019) (little cigars wrapper)—sequencing \\
\hline Firmicutes & Lysinibacillus & & $\begin{array}{l}\text { Chopyk et al. (2017a, b) (commercial cigarettes) - sequencing; Tyx et al. } 2020 \\
\text { (smokeless tobacco) - sequencing }\end{array}$ \\
\hline Firmicutes & Oceanobacillus & O. caeni & Han et al. (2016) (smokeless tobacco)—sequencing \\
\hline \multirow[t]{3}{*}{ Firmicutes } & Paenibacillus & P. amylolyticus & Chopyk et al. (2017a, b) (commercial cigarettes)—sequencing \\
\hline & & P. montaniterrae & Chopyk et al. $(2017 \mathrm{a}, \mathrm{b})$ (commercial cigarettes)—sequencing \\
\hline & & P. barengoltzii & $\begin{array}{l}\text { Malayil et al. (2020) (commercial cigarettes) - sequencing; Chattopadhyay et al. } \\
\text { (2019) (little cigars and cigarillos) - sequencing }\end{array}$ \\
\hline Firmicutes & Saccharibacillus & S. kuerlensis & Malayil et al. (2020) (commercial tobacco)—sequencing \\
\hline \multirow[t]{6}{*}{ Firmicutes } & Staphylococcus & S. sciuri & $\begin{array}{l}\text { Sapkota et al. (2010) (commercial cigarettes) - microarray, cloning, and sequencing; } \\
\text { Chattopadhyay et al. (2019) (little cigars and cigarillos) - sequencing }\end{array}$ \\
\hline & & S. aureus & Dygert (1957) (smokeless tobacco) - culture \\
\hline & & S. equorum & $\begin{array}{l}\text { Malayil et al. (2020) (commercial cigarettes) - sequencing; Chattopadhyay et al. } \\
\text { (2019) (little cigars and cigarillos) - sequencing }\end{array}$ \\
\hline & & S. saprophyticus & Sapkota et al. (2010) (commercial cigarettes) - microarray, cloning, and sequencing \\
\hline & & S. epidermis & $\begin{array}{l}\text { Sapkota et al. (2010) (commercial cigarettes)-microarray, cloning, and sequencing; } \\
\text { Dygert (1957) (smokeless tobacco)—culture }\end{array}$ \\
\hline & & S. cohnii & $\begin{array}{l}\text { Sapkota et al. (2010) (commercial cigarettes) - microarray, cloning, and sequencing; } \\
\text { Smyth et al. (2017) (smokeless tobacco)- sequencing }\end{array}$ \\
\hline Firmicutes & Sporosarcina & & Chopyk et al. $(2017 \mathrm{a}, \mathrm{b})$ (commercial cigarettes)—sequencing \\
\hline Firmicutes & Solibacillus & & Chopyk et al. $(2017 \mathrm{a}, \mathrm{b})$ (commercial cigarettes)—sequencing \\
\hline Firmicutes & Tetragenococcus & T. halophilus & $\begin{array}{l}\text { Han et al. (2016) (smokeless tobacco) - sequencing; Chattopadhyay et al. (2019) (little } \\
\text { cigars and cigarillos) - sequencing }\end{array}$ \\
\hline
\end{tabular}
cigars and cigarillos) — sequencing 
Table 1 (continued)

\begin{tabular}{|c|c|c|c|}
\hline Phylum & Genus & Species & Studies, products tested, and methods \\
\hline & & T. osmophilus & Smyth et al. (2019) (little cigars and cigarillos) - sequencing \\
\hline Firmicutes & Terribacillus & & Malayil et al. (2020) (commercial tobacco)—sequencing \\
\hline Firmicutes & Ureibacillus & & Chattopadhyay et al. (2019) (little cigars and cigarillos) - sequencing \\
\hline Firmicutes & Vagococcus & & Chopyk et al. $(2017 \mathrm{a}, \mathrm{b})$ (commercial cigarettes) — sequencing \\
\hline Firmicutes & Fonticella & & Smyth et al. (2017) (smokeless tobacco)—sequencing \\
\hline Proteobacteria & Acetobacter & & Smyth et al. (2017) (smokeless tobacco)—sequencing \\
\hline \multirow[t]{4}{*}{ Proteobacteria } & Acinetobacter & A. baumannii & Chopyk et al. (2017) (commercial cigarettes)—sequencing \\
\hline & & A. calcoaceticus & $\begin{array}{l}\text { Larsson et al. (2008) (commercial cigarettes) - sequencing; Chopyk et al. (2017a, } \\
\text { b) (commercial cigarettes) - sequencing }\end{array}$ \\
\hline & & A. rhizosphaerae & $\begin{array}{l}\text { Malayil et al. (2020) (commercial cigarettes); Chattopadhyay et al. (2019) } \\
\text { (little cigars and cigarillos)-sequencing }\end{array}$ \\
\hline & & A. schindleri & $\begin{array}{l}\text { Malayil et al. (2020) (commercial cigarettes) — sequencing; Chattopadhyay et al. } \\
\text { (2019) (little cigars and cigarillos) - sequencing }\end{array}$ \\
\hline Proteobacteria & Achromobacter & & Chopyk et al. $(2017 \mathrm{a}, \mathrm{b})$ (commercial cigarettes) - sequencing \\
\hline Proteobacteria & Aeromonas & & Chopyk et al. $(2017 \mathrm{a}, \mathrm{b})$ (commercial cigarettes) - sequencing \\
\hline Proteobacteria & Agrobacterium & A. tumefaciens & Chopyk et al. $(2017 \mathrm{a}, \mathrm{b})$ (commercial cigarettes)—sequencing \\
\hline Proteobacteria & Aurantimonas & A. altamirensis & Sapkota et al. (2010) (commercial cigarettes) — microarray, cloning, and sequencing \\
\hline Proteobacteria & Azospirillum & A. irakense & Chopyk et al. $(2017 \mathrm{a}, \mathrm{b})$ (commercial cigarettes)—sequencing \\
\hline Proteobacteria & Bacteriovorax & & Zhao et al. (2007) (flue cured tobacco leaves) - 16S rDNA PCR-DGGE technology \\
\hline Proteobacteria & Burkholderia & & $\begin{array}{l}\text { Sapkota et al. (2010) (commercial cigarettes) - microarray, cloning, and sequencing; } \\
\text { Smyth et al. (2017) (smokeless tobacco)-sequencing }\end{array}$ \\
\hline Proteobacteria & Campylobacter & & Sapkota et al. (2010) (commercial cigarettes)-microarray, cloning, and sequencing \\
\hline Proteobacteria & Comamonas & C. testosteroni & Sapkota et al. (2010) (commercial cigarettes)-microarray, cloning, and sequencing \\
\hline Proteobacteria & Cedecea & & Chopyk et al. (2017a, b) (commercial cigarettes)—sequencing \\
\hline Proteobacteria & Escherichia & E. coli & $\begin{array}{l}\text { Sapkota et al. (2010) (commercial cigarettes) - microarray, cloning, and sequencing; } \\
\text { Haque et al. (2017) (smokeless tobacco)-sequencing }\end{array}$ \\
\hline \multirow[t]{2}{*}{ Proteobacteria } & Enterobacter & E. amnigenus & Larsson et al. (2008) (commercial cigarettes) - culture \\
\hline & & E. cancerogenus & Larsson et al. (2008) (commercial cigarettes) - culture \\
\hline \multirow[t]{3}{*}{ Proteobacteria } & Erwinia & E. chrysanthemi & Chopyk et al. $(2017 \mathrm{a}, \mathrm{b})$ (commercial cigarettes) - sequencing \\
\hline & & E. agglomerans & Malayil et al. (2020) (commercial tobacco)—sequencing \\
\hline & & E. dispersa & $\begin{array}{l}\text { Malayil et al. (2020) (commercial cigarettes) — sequencing; Chattopadhyay et al. } \\
\text { (2019) (little cigars and cigarillos) - sequencing }\end{array}$ \\
\hline Proteobacteria & Gallibacterium & & Smyth et al. (2017) (smokeless tobacco)-sequencing \\
\hline Proteobacteria & Halomonas & & Han et al. (2016) (smokeless tobacco)—sequencing \\
\hline Proteobacteria & Herbaspirillum & H. huttiense & Smyth et al. (2019) (little cigars and cigarillos)-sequencing \\
\hline Proteobacteria & Klebsiella & K. oxytoca & Sapkota et al. (2010) (commercial cigarettes) - microarray, cloning, and sequencing \\
\hline Proteobacteria & Leptothrix & & Chattopadhyay et al. (2019) (little cigars wrapper)-sequencing \\
\hline Proteobacteria & Massilia & & $\begin{array}{l}\text { Chopyk et al. }(2017 \mathrm{a}, \mathrm{b}) \text { (commercial cigarettes) - sequencing; Tyx et al. (2020) } \\
\text { (smokeless tobacco) - sequencing }\end{array}$ \\
\hline Proteobacteria & Methylobacterium & M. adhaesivum & $\begin{array}{l}\text { Malayil et al. (2020) (commercial tobacco) - sequencing; Sapkota et al. (2010) (com- } \\
\text { mercial cigarettes) - microarray, cloning, and sequencing }\end{array}$ \\
\hline Proteobacteria & Novosphingobium & N. aromaticivorans & Sapkota et al. (2010) (commercial cigarettes) - microarray, cloning, and sequencing \\
\hline Proteobacteria & Pantoea & P agglomerans & $\begin{array}{l}\text { Larsson et al. (2008) (commercial cigarettes)—culture; Chattopadhyay et al. (2019) } \\
\text { (little cigars and cigarillos)—sequencing }\end{array}$ \\
\hline Proteobacteria & Pectobacterium & P. carotovorum & Chopyk et al. (2017a, b) (commercial cigarettes)—sequencing \\
\hline \multirow[t]{2}{*}{ Proteobacteria } & Proteus & P. mirabilis & Chopyk et al. $(2017 \mathrm{a}, \mathrm{b})$ (commercial cigarettes)—sequencing \\
\hline & & P.vulgaris & Dygert (1957) (smokeless tobacco)—culture \\
\hline
\end{tabular}


Table 1 (continued)

\begin{tabular}{|c|c|c|c|}
\hline Phylum & Genus & Species & Studies, products tested, and methods \\
\hline \multirow[t]{11}{*}{ Proteobacteria } & \multirow[t]{11}{*}{ Pseudomonas } & P. aeruginosa & $\begin{array}{l}\text { Dygert (1957) (smokeless tobacco)—culture; Sapkota et al. (2010) } \\
\text { (commercial cigarettes)-microarray, cloning, and sequencing }\end{array}$ \\
\hline & & P. stutzeri & Sapkota et al. (2010) (commercial cigarettes) - microarray, cloning, and sequencing \\
\hline & & P. fulva & Chopyk et al. $(2017 \mathrm{a}, \mathrm{b})$ (commercial cigarettes)—sequencing \\
\hline & & P. oryzihabitans & Chopyk et al. (2017a, b) (commercial cigarettes)—sequencing \\
\hline & & P. putida & Chopyk et al. $(2017 \mathrm{a}, \mathrm{b})$ (commercial cigarettes) - sequencing \\
\hline & & P. pseudoalcaligenes & $\begin{array}{l}\text { Malayil et al. (2020) (commercial cigarettes) - sequencing; Chattopadhyay et al. } \\
\text { (2019) (little cigars and cigarillos)—sequencing }\end{array}$ \\
\hline & & P. viridiflava & $\begin{array}{l}\text { Malayil et al. (2020) (commercial cigarettes) - sequencing; Chattopadhyay et al. } \\
\text { (2019) (little cigars and cigarillos) - sequencing }\end{array}$ \\
\hline & & P. veronii & Malayil et al. (2020) (commercial tobacco)—sequencing \\
\hline & & P. chlororaphis & Sapkota et al. (2010) (commercial cigarettes) - microarray, cloning, and sequencing \\
\hline & & P. cichorii & Sapkota et al. (2010) (commercial cigarettes) — microarray, cloning, and sequencing \\
\hline & & P. thermotolerans & Chattopadhyay et al. (2019) (little cigars wrapper)—sequencing \\
\hline Proteobacteria & Petrobacter & P. succinatimandens & Chattopadhyay et al. (2019) (little cigars wrapper) — sequencing \\
\hline Proteobacteria & Pseudoxanthomonas & \multirow[t]{3}{*}{ P. taiwanensis } & Chattopadhyay et al. (2019) (little cigars wrapper)—sequencing \\
\hline Proteobacteria & Ralstonia & & Smyth et al. (2017) (smokeless tobacco)—sequencing \\
\hline Proteobacteria & Salmonella & & Haque et al. (2017) (smokeless tobacco)—sequencing \\
\hline Proteobacteria & Sphingomonas & \multirow[t]{3}{*}{ S. multivorum } & Malayil et al. (2020) (commercial tobacco)—sequencing \\
\hline Proteobacteria & Schlegelella & & Chopyk et al. (2017a, b) (commercial cigarettes)—sequencing \\
\hline Proteobacteria & Silanimonas & & Chopyk et al. $(2017 \mathrm{a}, \mathrm{b})$ (commercial cigarettes) - sequencing \\
\hline Proteobacteria & Stenotrophomonas & \multirow[t]{2}{*}{ S. maltophilia } & $\begin{array}{l}\text { Sapkota et al. (2010) (commercial cigarettes) - microarray, cloning, and sequencing; } \\
\text { Chopyk et al. (2017a, b) (commercial cigarettes)-sequencing }\end{array}$ \\
\hline Proteobacteria & Serratia & & Sapkota et al. (2010) (commercial cigarettes) - microarray, cloning, and sequencing \\
\hline Proteobacteria & Shewanella & \multirow[t]{2}{*}{ S. algae } & Chattopadhyay et al. (2019) (little cigars and cigarillos) — sequencing \\
\hline Proteobacteria & Vibrio & & Haque et al. (2017) (smokeless tobacco)—sequencing \\
\hline Proteobacteria & Xanthomonas & \multirow[t]{2}{*}{ X. axonopodis } & Malayil et al. (2020) (commercial tobacco)—sequencing \\
\hline Proteobacteria & Paracoccus & & Sapkota et al. (2010) (commercial cigarettes) - microarray, cloning, and sequencing \\
\hline
\end{tabular}

in a lit cigarette and be transferred from the unburnt region of these products to the upper respiratory system via mainstream smoke, potentially influencing respiratory health. Ongoing studies in our lab provide evidence of viable bacteria in both tobacco products and the mainstream smoke of cigarettes, through the use of both culture-based approaches and DNA labeling coupled with sequencing (manuscript under review). For example, Bacillus, Paenibacillus, Terribacillus, and Desulfotomaculum have been isolated from mainstream cigarette smoke extract, indicating that viable tobacco-associated bacteria can potentially be transferred to the oral cavity via smoke particles (manuscript under review).

With reductions in cigarette smoking over the past 50 years (CDC 2019), the use of cigars, little cigars, and cigarillos have gone up in the USA (CDC Tobacco Free 2018). Although the chemical constituents of these alternative products have not been studied extensively, recent studies (Klupinski et al.
2016) identified more than 5000 chemicals in the mainstream smoke from the top-selling little cigars in the USA. To our knowledge, there are only a couple of studies that have focused on detailed characterization of bacterial communities found in little cigars' tobacco. Chattopadhyay et al. (2019) and Smyth et al. (2019) identified the predominant bacterial species as Lactobacillus, Bacillus, Staphylococcus, and Pseudomonas. Moreover, Chattopadhyay's study revealed that bacterial species significantly differed between that in the tobacco product and the wrapper. While the cigar wrapper was predominated by Lactobacillus and Bacillus, cigar tobacco had a higher relative abundance of Pseudomonas and Staphylococcus. Several potential pathogens were also identified in the little cigars: Pseudomonas pseudoalcaligenes and Staphylococcus sciuri in cigar tobacco and Pantoea agglomerans, Shewanella algae, and Acinetobacter schindleri in the wrappers. 


\section{Effect of mentholation}

In accordance with the "Special Rule for Cigarettes" in 2009, FDA now has the authority to ban flavors in traditional cigarettes that allure the younger population, such as strawberry, vanilla, and cinnamon, but these flavors still remain largely available in the market for other tobacco products like cigars, hookah, and e-cigarettes (U.S. FDA2020b). While no study has looked into the effects of flavoring on bacterial communities in hookah tobacco and e-liquids, data from our lab demonstrate a significant effect of flavors on the bacterial microbiome of the top two brands of hookah (manuscript under review). In 2018, FDA stated that it will seek to ban the use of menthol in combustible tobacco products, but to date, no such step has been taken by FDA to curtail the sale and use of menthol and tobacco flavors in tobacco products (Commissioner 2020).

Menthol is a simple monoterpene, used widely in tobacco manufacturing to create a cooling sensation, thereby reducing the harshness of tobacco smoke for a more pleasing smoking experience. Antimicrobial properties of menthol have been shown to inhibit a number of human and plant pathogenic bacteria (Ișcan et al. 2002). Recent research on the effects of mentholation on the cigarette tobacco microbiome shows a significant difference in overall bacterial diversity in mentholated tobacco compared to its non-menthol counterpart, but these differences are brand specific and are characterized by temporal shifts (Chopyk et al. 2017b; Malayil et al. 2020). While in some brands (e.g., Camel King), non-mentholated tobacco showed significantly higher bacterial diversity compared to "user-mentholated" tobacco (Chopyk et al. 2017a), brands like Newport and Marlboro showed higher or similar bacterial diversity in the commercially mentholated product type when compared to non-mentholated product types (Malayil et al. 2020). One explanation that Chopyk et al. (2017a, b) provide for this finding is that the degree and rate of menthol exposure in "user-mentholated" products vary from that in commercially mentholated products. This research also points out that mentholated products tend to harbor bacterial genera that are able to persist in harsh environments such as Anoxybacillus and Deinococcus. From these studies, other bacterial genera that were shown to be present at a significantly higher relative abundance in mentholated tobacco compared to non-mentholated tobacco were Thermus, Vagococcus, Silanimonas, Schlegelella, Sphingobacterium multivorum, Bacillus clausii, B. flexus, Methylobacterium adhaesivum, Erwinia dispersa, Xanthomonas axonopodis, and Acinetobacter schindleri.

Similar effects of mentholation were observed in little cigars. Chattopadhyay et al. (2019) compared Cheyenne full flavor non-menthol tobacco to Cheyenne menthol box and found significant decreases in bacterial diversity in both the tobacco and wrapper of the mentholated Cheyenne menthol box compared to the non-mentholated Cheyenne full flavor product. Specifically, menthol tobacco showed a significantly higher relative abundance of Ureibacillus, Lactobacillus, Bacillus, Corynebacterium, and Brachybacterium when compared to non-menthol tobacco.

\section{Water pipe/hookah}

Unlike cigarettes, little cigars, and cigarillos, the process by which the tobacco is burned is different in water pipe/hookah/ shisha smoking. Since tobacco smoke passes through a water bowl before being inhaled by the user, the majority of hookah smokers consider this process to be less harmful to their lungs than smoking a traditional combustible tobacco product (Schubert et al. 2011). Given the unique nature of smoke inhalation through a water pipe/hookah, very few studies have focused on the bacteria that might be present in the hookah tobacco products. There are several studies that have looked at the bacterial diversity in the hookah apparatus like the water bowl and mouthpiece (Safizadeh et al. 2014; Javadi et al. 2016). Hani et al. (2018) compared three different shisha tobacco products along with the various parts of the whole smoking apparatus. The authors reported some pathogenic bacteria (Halomonas, Staphylococcus, and Pseudomonas) and less than $1 \%$ of the gut commensal, Faecalibacterium, as well as Streptophyta, Shewanella, and Propionibacterium, in shisha tobacco (Hani et al. 2018). The two major studies that have evaluated the presence of bacteria in hookah tobacco have identified several bacterial species including Streptophyta, Halomonas, Pseudomonas viridiflava, Paenibacillus lautus, Propionibacterium acnes, Staphylococcus, Shewanella, Novosphingobium, Sphingomonas multivorum, Faecalibacterium, Methylobacterium adhaesivum, Flavobacterium, Bacillus cereus, B. clausii, B. flexus, Terribacillus, Janthinobacterium, Agrobacterium, and Variovorax paradoxus (Hani et al. 2018) (manuscript under review).

\section{Smokeless tobacco/chewing tobacco}

Unlike combustible tobacco products, smokeless tobacco (e.g., chewing tobacco, snuff, snus, dissolvable tobacco, and dip) is chewed, sniffed, or sucked by the user in order to absorb nicotine. The impacts of smokeless tobacco have more significantly been studied with regard to the development of oral cancers, oral lesions, and other related dental diseases, with less focus on the microbial communities residing within these products.

However, culture studies have identified Bacillus megaterium, B. pumilus, B. brevis, B. licheniformis, and $B$. subtilis in popular USA chewing tobacco brands (Rubinstein and Pedersen 2002; Han et al. 2016). Han et al. (2016) also demonstrated the presence of Oceanobacillus, 
Staphylococcus, and Tetragenococcus through culture work. But very few studies have considered the bacterial diversities of smokeless tobacco products using next-generation sequencing technologies. Tyx et al. (2016) compared U.S. dry and moist snuff products with Swedish snus and Sudanese toombak using Ion Torrent PGM (Thermo Fisher Scientific Inc.; Waltham, MA) sequencing. Bacillus, Corynebacterium, Staphylococcus, Pseudomonas, and Tetragenococcus dominated the tested smokeless tobacco products (Tyx et al. 2016). A similar study by Smyth et al. (2017) using 454 pyrosequencing found that the prevalent bacterial phyla in smokeless tobacco products were Firmicutes, Proteobacteria, Actinobacteria, and Bacteroidetes. Both studies showed dry snuff products to harbor higher bacterial diversity than moist snuff and Swedish snus. Additional species identified included Acetobacter, Burkholderia, and Streptomyces (Smyth et al. 2017) and Tetragenococcus, Carnobacterium, Lactobacillus, Geobacillus, Bacillus, and Staphylococcus (Han et al. 2016). Al-Hebshi et al. (2017) compared bacterial communities in Swedish snus and Yemeni shammah. Tyx et al. (2020) identified a high relative abundance of Bacillus, Paenibacillus, and Oceanobacillus in American snuff and Pseudomonas, Massilia, Propionibacterium, Puniceispirillum, and Gloeothece in Swedish snus. While Sudanese toombak had a higher relative abundance of Facklamia, Desemzia, Atopostipes, and Lysinibacillus, and Yemeni shammah exclusively contained Bacillus (Al-hebshi et al. 2017).

Other common forms of chewing tobacco in Southeast Asia are betel leaf (commonly known as paan), gutka, khaini, and zarda. Culture studies have identified Escherichia coli, Salmonella, Vibrio, Bacillus, and Staphylococcus from paan (Haque et al. 2017), Klebsiella from gutka, and Bacillus from khaini and zarda (Shetty and Hegde 2015; Mehra et al. 2020). A South African pilot study on local smokeless tobacco products also showed the presence of Bacillus in three traditional tobacco mixes and two industry-produced tobacco mixes (Taxi and Ntsu) (Ayo-Yusuf et al. 2005).

\section{Electronic cigarettes}

Unlike conventional tobacco products, for which FDA mandates that health warnings must be placed on products, there is no clear regulation or warnings on e-cigarette packaging, and their long-term use and resulting health effects remain largely unknown. Subsequently, there are limited studies concerning the microbiomes of e-cigarette liquids and cartridges. Although no studies show direct evidence of the presence of inflammatory bacterial endotoxin or fungi in e-liquids, a recent study (Lee et al. 2019) detected the presence of bacterial endotoxin and fungal cell wall glucan in $23 \%$ and $81 \%$ of the tested products, respectively. Endotoxin concentrations were found to be 3.2 times higher in the cartridges than that in the e-liquids.
Glucan concentrations in tobacco and menthol flavored eliquids were 10 and 3.5 times higher than that in fruit flavored e-liquids, respectively. Gilpin et al. (2019) demonstrated a significant effect of e-cigarette vapor on lung pathogens' biofilm formation and cytokine secretion, potentially increasing lung inflammation. E-cigarette smoker's oral microbiome also showed significantly higher bacterial OTUs (species) and significantly different bacterial communities when compared to that in nonsmokers (Pushalkar et al. 2020). The bacterial taxa in the saliva were closely associated with the nicotine intake among the e-cigarette users, and oral bacterial infection was significantly accelerated with exposure to e-cigarette aerosols.

\section{Fungal constituents of tobacco products}

Unlike bacteria, a detailed characterization of the fungal community in tobacco products is almost completely lacking. As early as the 1970s, researchers have been studying the fungal microbiome of tobacco products (Table 2). Early culture study in cigarettes (Papavassiliou et al. 1971) showed the presence of Aspergillus, Penicillium, Mucor, Alternaria, Cladosporium, Streptomyces, Candida, Geotrichum, Cephalosporium, and Scopulariopsis. Strains of Aspergillus were isolated from commercial cigarettes and pipe tobacco pointing towards the potential allergenic complications developing from smoking these products (Kurup et al. 1983). Studies on Nigerian cigarettes showed the presence of thermophilic fungal pathogens such as Thermoascus aurantiacus, Mucor pusillus, and M. miehei, in addition to Chaetomium thermophile, Humicola insolens, H. lanuginosa, Malbranchea pulchella, Talaromyces duponti, and Thermoascus crustaceus (Ogundero 1980). A total of 42 species including Aspergillus flavus, A. flavus var. columnaris, A. fumigatus, A. niger, Penicillium chrysogenum, $P$. funiculosum, Fusarium moniliforme, F. oxysporum, and $F$. solani were identified through culture work in cigarettes from Egypt (El-Maghraby and Abdel-Sater 1993). Fungal contamination with A. fumigatus, Fusarium, Acremonium, Rhizopus, and Scedosporium were identified in 14 brands of cigarettes but were absent in the cigarette smoke (Verweij 2000). The presence of ergosterol (a biomarker for fungal biomass) was detected in cigarettes, and an increase in concentration was observed when the cigarettes were stored under different humidity conditions (Larsson et al. 2008).

Early culture studies on 14 snuff products in the USA also showed the presence of thermophilic Humicola lanuginosa, Thielavia albomyces, M. pulchella var. sulfurea, and Talaromyces thermophilus growing from three of the tested products (Tansey 1975). Aspergillus, Penicillium, Mucor, Sepedonium, and Trichophyton were present in 600 samples of smokeless tobacco products collected from Pakistan (Saleem et al. 2018). Meanwhile, a culture study and identification based on morphology identified Aspergillus fumigatus, 
Table 2 A list of culture-based studies describing fungal profiles in tobacco products

\begin{tabular}{|c|c|c|c|}
\hline Division & Genera & Species & Studies and products tested \\
\hline \multirow[t]{4}{*}{ Ascomycota } & \multirow[t]{4}{*}{ Aspergillus } & A. fumigatus & $\begin{array}{l}\text { Papavassiliou et al. (1971) (commercial cigarettes); Larsson et al. (2008) (fresh leaves, commercial } \\
\text { cigarettes, and smoke); Kurup et al. (1983) (commercial cigarettes); } \\
\text { El-Maghraby and Abdel-Sater (1993) (commercial cigarettes); Verweij et al. (2000) (commercial } \\
\text { cigarettes); Shetty and Hegde (2015) (smokeless tobacco) }\end{array}$ \\
\hline & & A. flavus & Welty (1972) (flue cured tobacco); El-Maghraby and Abdel-Sater (1993) (commercial cigarettes) \\
\hline & & A. niger & Welty (1972) (flue cured tobacco); El-Maghraby and Abdel-Sater (1993) (commercial cigarettes) \\
\hline & & A. repens & Welty (1972) (flue cured tobacco) \\
\hline \multirow[t]{2}{*}{ Ascomycota } & \multirow[t]{2}{*}{ Alternaria } & & $\begin{array}{l}\text { Bogden et al. (1981) (commercial cigarettes); Forgacs and Carll (1966) (commercial cigarettes, } \\
\text { cigars, and pipe tobacco) }\end{array}$ \\
\hline & & A. alternata & Welty (1972) (flue cured tobacco) \\
\hline Ascomycota & Acremonium & & Verweij et al. (2000) (commercial cigarettes) \\
\hline \multirow[t]{3}{*}{ Ascomycota } & \multirow[t]{3}{*}{ Penicillium } & P. cyclopium & Welty (1972) (flue cured tobacco) \\
\hline & & P. chrysogenum & El-Maghraby and Abdel-Sater (1993) (commercial cigarettes) \\
\hline & & P. funiculosum & El-Maghraby and Abdel-Sater (1993) (commercial cigarettes) \\
\hline \multirow[t]{2}{*}{ Zygomycota } & \multirow[t]{2}{*}{ Mucor } & M. pusillus & Ogundero (1980) (commercial cigarettes, cured tobacco leaves) \\
\hline & & M. miehei & Ogundero (1980) (commercial cigarettes, cured tobacco leaves) \\
\hline Ascomycota & Cladosporium & & Papavassiliou et al. (1971) (commercial cigarettes) \\
\hline Ascomycota & Geotrichum & & Papavassiliou et al. (1971) (commercial cigarettes) \\
\hline Ascomycota & Scopulariopsis & & Papavassiliou et al. (1971) (commercial cigarettes) \\
\hline Ascomycota & Chaetomium & C. thermophile & Ogundero (1980) (commercial cigarettes, cured tobacco leaves) \\
\hline \multirow[t]{2}{*}{ Ascomycota } & \multirow[t]{2}{*}{ Humicola } & H. insolens & Ogundero (1980) (commercial cigarettes, cured tobacco leaves) \\
\hline & & H. lanuginosa & Ogundero (1980) (commercial cigarettes, cured tobacco leaves); Tansey (1975) (smokeless tobacco) \\
\hline Ascomycota & Malbranchea & M. pulchella & Ogundero (1980) (commercial cigarettes, cured tobacco leaves); Tansey (1975) (smokeless tobacco) \\
\hline \multirow[t]{2}{*}{ Ascomycota } & \multirow[t]{2}{*}{ Talaromyces } & T. duponti & Ogundero (1980) (commercial cigarettes, cured tobacco leaves) \\
\hline & & T. thermophilus & Tansey (1975) (smokeless tobacco) \\
\hline \multirow[t]{2}{*}{ Ascomycota } & \multirow[t]{2}{*}{ Thermoascus } & T. crustaceus & Ogundero (1980) (commercial cigarettes, cured tobacco leaves) \\
\hline & & T. aurantiacus & Ogundero (1980) (commercial cigarettes, cured tobacco leaves) \\
\hline \multirow[t]{3}{*}{ Ascomycota } & \multirow[t]{3}{*}{ Fusarium } & F. moniliforme & El-Maghraby and Abdel-Sater (1993) (commercial cigarettes) \\
\hline & & F. oxysporum & El-Maghraby and Abdel-Sater (1993) (commercial cigarettes) \\
\hline & & F. solani & El-Maghraby and Abdel-Sater (1993) (commercial cigarettes) \\
\hline Mucoromycota & Rhizopus & & Verweij et al. (2000) (commercial cigarettes) \\
\hline Ascomycota & Scedosporium & & Verweij et al. (2000) (commercial cigarettes) \\
\hline Ascomycota & Thielavia & T. albomyces & Tansey (1975) (smokeless tobacco) \\
\hline Ascomycota & Sepedonium & & Saleem et al. (2018) (smokeless tobacco) \\
\hline Ascomycota & Trichophyton & & Saleem et al. (2018) (smokeless tobacco) \\
\hline
\end{tabular}

along with Bacillus and Klebsiella in gutka (Shetty and Hegde 2015).

\section{Conclusions and future directions}

In the past decade, the tobacco microbiome has received increasing scientific attention, and multiple studies have characterized the bacterial communities that colonize a variety of smoked and smokeless tobacco products. Nevertheless, there exist gaps in our understanding of the complex bacterial communities in these products, as well as their potential adverse effects on users' oral and lung microbiomes and overall health.

One of the most important questions that require additional research is whether or not these bacteria are viable and are able to transfer to the user's respiratory tract via mainstream smoke. Another area that remains largely unexplored is the fungal communities present in tobacco products. Multiple fungal genera are known human pathogens that are responsible for causing oral and lung associated diseases.

In addition, there is a lack of research focused on characterizing the potential microbiome of electronic liquids. 
Multiple studies have focused on the physical and chemical constituents of these liquids and cartridges. Previous studies have shown that flavors or additives in tobacco products have significantly affected bacterial diversity. However, there is a lack of data on the effects of the multiple flavors of hookah that are available in the market on the tobacco microbiome. Specifically, the effects of mint and menthol remain to be explored.

Studying the viral and protozoan communities of tobacco products has also been largely ignored. However, of note, a recent study (de Bernardis and Busà 2020) suggested the role of tobacco mosaic virus (TMV) as an immunological mediator for resistance against the SARS-CoV-2 virus. Given the current global pandemic crisis, it may be even more important than ever to study the potential viral communities of tobacco products, as well as their effects on users' overall health.

Authors' contributions SC and LM conducted the literature review, summarized the findings, wrote the first draft of the manuscript, developed the tables and figure, and edited and finalized the manuscript. EFM and ARS conceived the manuscript idea, supervised the process, edited the manuscript, and approved the final version. ARS served as the corresponding author.

Funding The work in the Sapkota laboratory related to this mini-review topic was funded by grant number P50CA180523 from the National Cancer Institute and FDA Center for Tobacco Products (CTP) awarded to the University of Maryland.

\section{Declarations}

Ethics approval This article does not contain any studies with human participants or animals performed by any of the authors.

Conflict of interest The authors declare that they have no conflict of interest.

\section{References}

Al-hebshi NN, Alharbi FA, Mahri M, Chen T (2017) Differences in the bacteriome of smokeless tobacco products with different oral carcinogenicity: compositional and predicted functional analysis. Genes 8:106. https://doi.org/10.3390/genes8040106

Ayo-Yusuf OA, van Wyk C, van Wyk CW, de Wet I (2005) Smokeless tobacco products on the south African market do not inhibit oral bacterial flora: a pilot study. South Afr J Epidemiol Infect 20:136-139. https://doi.org/10.1080/ 10158782.2005.11441251

Bogden JD, Kemp FW, Buse M, Thind IS, Louria DB, Forgacs J, Llanos G, Moncoya Terrones I (1981) Composition of tobaccos from countries with high and low incidences of lung cancer. I. selenium, polonium-210, alternaria, tar, and nicotine. JNCI: Journal of the National Cancer Institute 66:27-31. https://doi.org/10.1093/jnci/ 66.1 .27

(2019) Cigarette Smoking Among U.S. Adults Hits All-Time Low | CDC Online Newsroom | CDC. https://www.cdc.gov/media/releases/ 2019/p1114-smoking-low.html. Accessed 9 Nov 2020
CDC Tobacco Free (2018) 2014 SGR: the health consequences of smoking - 50 years of progress. In: Centers for Disease Control and Prevention. https://www.cdc.gov/tobacco/data_statistics/sgr/ 50th-anniversary/index.htm. Accessed 9 Nov 2020

CDC Tobacco Free (2020) Tobacco brand preferences. In: Centers for Disease Control and Prevention. https://www.cdc.gov/tobacco/ data_statistics/fact_sheets/tobacco_industry/brand_preference/ index.htm. Accessed 16 Nov 2020

Chattopadhyay S, Smyth EM, Kulkarni P, Babik KR, Reid M, Hittle LE, Clark PI, Mongodin EF, Sapkota AR (2019) Little cigars and cigarillos harbor diverse bacterial communities that differ between the tobacco and the wrapper. PLoS One 14:e0211705. https://doi.org/ 10.1371/journal.pone.0211705

Chopyk J, Chattopadhyay S, Kulkarni P, Claye E, Babik KR, Reid MC, Smyth EM, Hittle LE, Paulson JN, Cruz-Cano R, Pop M, Buehler SS, Clark PI, Sapkota AR, Mongodin EF (2017a) Mentholation affects the cigarette microbiota by selecting for bacteria resistant to harsh environmental conditions and selecting against potential bacterial pathogens. Microbiome 5:22. https://doi.org/10.1186/s40168017-0235-0

Chopyk J, Chattopadhyay S, Kulkarni P, Smyth EM, Hittle LE, Paulson JN, Pop M, Buehler SS, Clark PI, Mongodin EF, Sapkota AR (2017b) Temporal Variations in Cigarette Tobacco Bacterial Community Composition and TobaccoSpecific Nitrosamine Content Are Influenced by Brand and Storage Conditions Front Microbiol 08. https://doi.org/10. 3389/fmicb.2017.00358

Cigarette Smoking Among U.S. Adults Hits All-Time Low | CDC Online Newsroom | Centers for Disease Control and Prevention (2019) https://www.cdc.gov/media/releases/2019/p1114-smoking-low. html. Accessed 28 Feb 2021

Collins MD, Aguirre M, Facklam RR, Shallcross J, Williams AM (1992) Globicatella sanguis gen.nov., sp.nov., a new Gram-positive catalasenegative bacterium from human sources. J Appl Bacteriol 73:433-437. https://doi.org/10.1111/j.1365-2672.1992.tb05000.x

Commissioner O Of the (2020) statement from FDA commissioner scott Gottlieb, M.D., on proposed new steps to protect youth by preventing access to flavored tobacco products and banning menthol in cigarettes. In: FDA https://www.fda.gov/news-events/pressannouncements/statement-fda-commissioner-scott-gottlieb-mdproposed-new-steps-protect-youth-preventing-access. Accessed 9 Nov 2020

Creamer MR (2019) Tobacco product use and cessation indicators among adults - United States, 2018. MMWR Morb Mortal Wkly Rep 68. https://doi.org/10.15585/mmwr.mm6845a2

de Andrade M, Hastings G, Angus K (2013) Promotion of electronic cigarettes: tobacco marketing reinvented? BMJ 347. https://doi. org/10.1136/bmj.f7473

de Bernardis E, Busà L (2020) A putative role for the tobacco mosaic virus in smokers' resistance to COVID-19. Med Hypotheses 143: 110153. https://doi.org/10.1016/j.mehy.2020.110153

Dygert HP (1957) Snuff - A source of pathogenic bacteria in chronic bronchitis. N Engl J Med 257:311-313. https://doi.org/10.1056/ NEJM195708152570704

Eaton T, Falkinham JO, von Reyn CF (1995) Recovery of Mycobacterium avium from cigarettes. J Clin Microbiol 33:27572758

El-Maghraby OMO, Abdel-Sater MA (1993) Mycoflora and natural occurrence of mycotoxins in tobacco from cigarettes in Egypt. Zentralblatt für Mikrobiologie 148:253-264. https://doi.org/10. 1016/S0232-4393(11)80101-0

Erb-Downward JR, Thompson DL, Han MK, Freeman CM, McCloskey L, Schmidt LA, Young VB, Toews GB, Curtis JL, Sundaram B, Martinez FJ, Huffnagle GB (2011) Analysis of the lung microbiome in the "healthy" smoker and in COPD. PLoS One 6:e16384. https:// doi.org/10.1371/journal.pone. 0016384 
Fodor AA, Klem ER, Gilpin DF, Elborn JS, Boucher RC, Tunney MM, Wolfgang MC (2012) The adult cystic fibrosis airway microbiota is stable over time and infection type, and highly resilient to antibiotic treatment of exacerbations. PLoS One 7:e45001. https://doi.org/10. 1371/journal.pone.0045001

Forgacs J, Carll WT (1966) Mycotoxicoses: toxic fungi in tobaccos. Science 152:1634-1635. https://doi.org/10.1126/science.152.3729. 1634

Gilpin DF, McGown K-A, Gallagher K, Bengoechea J, Dumigan A, Einarsson G, Elborn JS, Tunney MM (2019) Electronic cigarette vapour increases virulence and inflammatory potential of respiratory pathogens. Respir Res 20:267. https://doi.org/10.1186/s12931-0191206-8

Götz F (2002) Staphylococcus and biofilms. Mol Microbiol 43:13671378. https://doi.org/10.1046/j.1365-2958.2002.02827.x

Han J, Sanad YM, Deck J, Sutherland JB, Li Z, Walters MJ, Duran N, Holman MR, Foley SL (2016) Bacterial populations associated with smokeless tobacco products. Appl Environ Microbiol 82:62736283. https://doi.org/10.1128/AEM.01612-16

Hani J, Abdel Nour G, Matta J, Jazzar B, Pfaffl MW, Hanna-Wakim L, Abdel Nour AM (2018) Shisha microbiota: the good, the bad and the not so ugly. BMC Res Notes 11:446. https://doi.org/10.1186/ s13104-018-3553-9

Haque MM, Sarker MAR, Rifa RA, Islam MA, Khatun MM (2017) Detection of food-borne bacteria in ready to eat betel leaf sold at local markets in Mymensingh. Vet World 10:1040-1045. https:// doi.org/10.14202/vetworld.2017.1040-1045

Tobacco | Healthy People 2020. https://www.healthypeople.gov/2020/ leading-health-indicators/2020-lhi-topics/Tobacco. Accessed 16 Nov 2020a

Huang R, Li M, Ye M, Yang K, Xu X, Gregory RL (2014) Effects of nicotine on Streptococcus gordonii growth, biofilm formation, and cell aggregation. Appl Environ Microbiol 80:7212-7218. https:// doi.org/10.1128/AEM.02395-14

Ișcan G, Kirimer N, Kürkcüoğlu M, Bașer KHC, Demirci F (2002) Antimicrobial screening of mentha piperita essential oils. J Agric Food Chem 50:3943-3946. https://doi.org/10.1021/ jf011476k

Javadi A, Sadr M, Shamaei M, Pourabdollah M, Arjmand M, Abdolahinia A (2016) Waterpipe tobacco smoking may potentiate risk of fungal and bacterial infections. Eur Respi J 48. https://doi. org/10.1183/13993003.congress-2016.PA4608

Kaelin P, Gadani F (2000) Occurrence of bacillus thuringiensis on cured tobacco leaves. Curr Microbiol 40(3):205-209. https://doi.org/10. $1007 / \mathrm{s} 002849910041$

Kaelin P, Morel P, Gadani F (1994) Isolation of Bacillus thuringiensis from stored tobacco and lasioderma serricorne (F.). Appl Environ Microbiol 60:19-25

Klupinski TP, Strozier ED, Friedenberg DA, Brinkman MC, Gordon SM, Clark PI (2016) Identification of new and distinctive exposures from little cigars. Chem Res Toxicol 29:162-168. https://doi.org/10. 1021/acs.chemrestox.5b00371

Konstantinou E, Fotopoulou F, Drosos A, Dimakopoulou N, Zagoriti Z, Niarchos A, Makrynioti D, Kouretas D, Farsalinos K, Lagoumintzis G, Poulas K (2018) Tobacco-specific nitrosamines: a literature review. Food Chem Toxicol 118:198-203. https://doi.org/10.1016/j. fct.2018.05.008

Kostygina G, Glantz SA, Ling PM (2016) Tobacco industry use of flavours to recruit new users of little cigars and cigarillos. Tob Control 25:66-74. https://doi.org/10.1136/tobaccocontrol-2014051830

Kreslake JM, Wayne GF, Alpert HR, Koh HK, Connolly GN (2008) Tobacco industry control of menthol in cigarettes and targeting of adolescents and young adults. Am J Public Health 98:1685-1692. https://doi.org/10.2105/AJPH.2007.125542
Kurup VP, Resnick A, Kagen SL, Cohen SH, Fink JN (1983) Allergenic fungi and Actinomycetes in smoking materials and their health implications. Mycopathologia 82:61-64. https://doi.org/10.1007/ BF00436948

Landau E, Shapira R (2012) Effects of subinhibitory concentrations of menthol on adaptation, morphological, and gene expression changes in Enterohemorrhagic Escherichia coli. Appl Environ Microbiol 78: 5361-5367. https://doi.org/10.1128/AEM.00894-12

Larsson L, Szponar B, Ridha B, Pehrson C, Dutkiewicz J, KrysińskaTraczyk E, Sitkowska J (2008) Identification of bacterial and fungal components in tobacco and tobacco smoke. Tob Induc Dis 4:4. https://doi.org/10.1186/1617-9625-4-4

Lee M-S, Allen JG, Christiani DC (2019) Endotoxin and $(1 \rightarrow 3)-\beta-D-$ glucan contamination in electronic cigarette products sold in the United States. Environ Health Perspect 127:047008. https://doi. org/10.1289/EHP3469

Malayil L, Chattopadhyay S, Kulkarni P, Hittle L, Clark PI, Mongodin EF, Sapkota AR (2020) Mentholation triggers brand-specific shifts in the bacterial microbiota of commercial cigarette products. Appl Microbiol Biotechnol 104:6287-6297. https://doi.org/10.1007/ s00253-020-10681-1

Mehra R, Mohanty V, Balappanavar AY, Kapoor S (2020) Bacterial contamination of packaged smokeless tobacco sold in India. Tob Prev Cessation 6: . https://doi.org/10.18332/tpc/115064

Ogundero VW (1980) Thermophilic mycoflora of cigarettes and cured tobacco leaves. Mycopathologia 71:9-11. https://doi.org/10.1007/ BF00625306

Papavassiliou J, Piperakis G, Marcelou-Kinti U (1971) Mycological flora of cigarettes. Mycopathol Mycol Appl 44:117-120. https://doi.org/ 10.1007/BF02051879

Patel T, Ishiuji Y, Yosipovitch G (2007) Menthol: a refreshing look at this ancient compound. J Am Acad Dermatol 57:873-878. https://doi. org/10.1016/j.jaad.2007.04.008

Pauly JL, Paszkiewicz G (2011) Cigarette smoke, Bacteria, Mold, microbial toxins, and chronic lung inflammation. In: J Oncol. https:// www.hindawi.com/journals/jo/2011/819129/. Accessed 27 Aug 2020

U.S. FDA (2020a) Section 904 of the Federal Food, drug, and cosmetic act - submission of health information to the secretary. FDA. https:// www.fda.gov/tobacco-products/rules-regulations-and-guidance/ section-904-federal-food-drug-and-cosmetic-act-submissionhealth-information-secretary

U.S. FDA (2020b) Section 907 of the Federal Food, drug, and cosmetic act - Tobacco product standards. FDA. https://www.fda.gov/ tobacco-products/rules-regulations-and-guidance/section-907federal-food-drug-and-cosmetic-act-tobacco-product-standards

Pushalkar S, Paul B, Li Q, Yang J, Vasconcelos R, Makwana S, González JM, Shah S, Xie C, Janal MN, Queiroz E, Bederoff M, Leinwand J, Solarewicz J, Xu F, Aboseria E, Guo Y, Aguallo D, Gomez C, Kamer A, Shelley D, Aphinyanaphongs Y, Barber C, Gordon T, Corby P, Li X, Saxena D (2020) Electronic cigarette aerosol modulates the oral microbiome and increases risk of infection. iScience 23:100884. https://doi.org/10.1016/j.isci. 2020.100884

Rooney AP, Swezey JL, Wicklow DT, McAtee MJ (2005) Bacterial species diversity in cigarettes linked to an investigation of severe pneumonitis in U.S. military personnel deployed in operation Iraqi freedom. Curr Microbiol 51:46-52. https://doi.org/10.1007/s00284005-4491-z

Rubinstein I, Pedersen GW (2002) Bacillus species are present in chewing Tobacco sold in the United States and evoke plasma exudation from the Oral mucosa. Clin Diagn Lab Immunol 9:10571060. https://doi.org/10.1128/CDLI.9.5.1057-1060.2002

Safizadeh H, Moradi M, Rad MR, Nakhaee N (2014) Bacterial contamination of different components of the waterpipe. Int $\mathrm{J}$ 
Tuberc Lung Dis 18:988-991. https://doi.org/10.5588/ijtld.14. 0026

Saleem S, Naz SA, Shafique M, Jabeen N, Ahsan SW (2018) Fungal contamination in smokeless tobacco products traditionally consumed in Pakistan. J Pak Med Assoc 68:1471-1477

Sapkota AR, Berger S, Vogel TM (2010) Human pathogens abundant in the bacterial metagenome of cigarettes. Environ Health Perspect 118:351-356. https://doi.org/10.1289/ehp.0901201

Schelz Z, Molnar J, Hohmann J (2006) Antimicrobial and antiplasmid activities of essential oils. Fitoterapia 77:279-285. https://doi.org/ 10.1016/j.fitote.2006.03.013

Schubert J, Kappenstein O, Luch A, Schulz TG (2011) Analysis of primary aromatic amines in the mainstream waterpipe smoke using liquid chromatography-electrospray ionization tandem mass spectrometry. J Chromatogr A 1218:5628-5637. https://doi.org/10. 1016/j.chroma.2011.06.072

Shapira R, Mimran E (2007) Isolation and characterization of Escherichia coli mutants exhibiting altered response to thymol. Microb Drug Resist 13:157-165. https://doi.org/10.1089/mdr.2007.731

Shetty PJ, Hegde V (2015) Assessment of pathogenic microorganisms in commercially available smokeless tobacco products. Avicenna J Med 5:54-55. https://doi.org/10.4103/22310770.154202

Smyth EM, Kulkarni P, Claye E, Stanfill S, Tyx R, Maddox C, Mongodin EF, Sapkota AR (2017) Smokeless tobacco products harbor diverse bacterial microbiota that differ across products and brands. Appl Microbiol Biotechnol 101:5391-5403. https://doi.org/10.1007/ s00253-017-8282-9

Smyth EM, Chattopadhyay S, Babik K, Reid M, Chopyk J, Malayil L, Kulkarni P, Hittle LE, Clark PI, Sapkota AR, Mongodin EF (2019) The bacterial communities of little cigars and cigarillos are dynamic over time and varying storage conditions. Front Microbiol 10. https://doi.org/10.3389/fmicb.2019.02371

Tansey MR (1975) Isolation of thermophilic fungi from snuff. Appl Microbiol 29:128-129
Tyx RE, Stanfill SB, Keong LM, Rivera AJ, Satten GA, Watson CH (2016) Characterization of bacterial communities in selected smokeless tobacco products using $16 \mathrm{~S}$ rDNA analysis. PLoS One 11:e0146939. https://doi.org/10.1371/journal.pone. 0146939

Tyx RE, Rivera AJ, Keong LM, Stanfill SB (2020) An exploration of smokeless tobacco product nucleic acids: a combined metagenome and metatranscriptome analysis. Appl Microbiol Biotechnol 104:751-763. https://doi.org/10.1007/s00253-01910232-3

Verweij PE (2000) Fungal contamination of tobacco and marijuana. JAMA 284:2875. https://doi.org/10.1001/jama.284.22.2869

Welty RE (1972) Fungi isolated from flue-cured tobacco sold in Southeast United States, 1968-1970. Appl Microbiol 24:518-520

WHO | WHO report on the global tobacco epidemic 2017. https://www. who.int/tobacco/global_report/2017/en/. Accessed 9 Nov 2020b

Wu Y, Ma Y, Xu T, Zhang Q, Bai J, Wang J, Zhu T, Lou Q, Götz F, Qu D, Zheng C, Zhao K (2018) Nicotine enhances Staphylococcus epidermidis biofilm formation by altering the bacterial autolysis, extracellular DNA releasing, and polysaccharide intercellular adhesin production. Front Microbiol 9. https://doi.org/10.3389/ fmicb.2018.02575

Zschiedrich CP, Keidel V, Szurmant H (2016) Molecular mechanisms of two-component signal transduction. J Mol Biol 428:3752-3775. https://doi.org/10.1016/j.jmb.2016.08.003

Zhao M, Wang B, Li F, Qiu L, Li F, Wang S, Cui J (2007) Analysis of bacterial communities on aging flue-cured tobacco leaves by $16 \mathrm{~S}$ rDNA PCR-DGGE technology. Appl Microbiol Biotechnol 73: 1435-1440. https://doi.org/10.1007/s00253-006-0625-x

Publisher's note Springer Nature remains neutral with regard to jurisdictional claims in published maps and institutional affiliations. 